\title{
Visualización de Desempeños Ambientales de Viviendas en BIM
}

Visualization of Housing Environmental Performances in BIM

\author{
Gerth Wandersleben \\ Universidad del Bío-Bío, Chile \\ gerthwwm@gmail.com \\ Rodrigo García Alvarado \\ Universidad del Bío-Bío, Chile \\ rgarcia@ubiobio.cl
}

\author{
Claudio Labarca Montoya \\ Pontificia Universidad Católica de Chile \\ clabarca@uc.cl
}

\author{
Cristian Muñoz Viveros \\ Universidad del Bío-Bío, Chile \\ cmunozv@ubiobio.cl
}

\begin{abstract}
In order to promote a sustainable design in building information modeling systems (BIM), it proposes a color-code to mark the volume and rooms according geometric relationships defined by parametric environmental simulations. For suggest the estimated performance of the building and sectors, and encourage design adjustments to reduce energy demands with proper comfort. This approach is applied to single housing in temperate-humid climate based on records and analysis carried on in Concepción, Chile. Like a visual aid for designers to improve environmental performance of buildings.
\end{abstract}

Keywords: Building Information Modeling (BIM); Building Performance Simulation (BPS); Integrated Design; Housing.

\section{Introducción}

El diseño sustentable requiere integrar consideraciones ambientales desde el comienzo del proyecto arquitectónico, en que se pueden adoptar mejoramientos sustanciales de forma, distribución o materialidad (Morbitzer et al, 2001). Mientras las adecuaciones posteriores suelen ser costosas y de menor incidencia.

Los proyectos arquitectónicos están utilizando creciente-mente plataformas BIM (Building Information Modeling). Las últimas versiones de estos programas están incorporando análisis ambientales, como Revit 14 con Green Building Studio y Archicad con Ecodesigner. También pueden exportar la información del diseño para revisar en un programa de simulación BPS (Building Performance Simulation), como Ecotect, Design Builder o TAS (Krieger, 2008). Sin embargo estos requieren la descripción completa del edificio y carecen de condiciones que orienten el diseño. Pueden efectuar un cálculo detallado del comportamiento ambiental del edificio, pero exigen un esfuerzo posterior $y$ particular al proyecto, cuando ya es difícil realizar modificaciones. Por otro lado se pueden establecer regulaciones o certificaciones generales, pero éstas son básicas y homogéneas. Presentando un constante dilema entre seguir un enfoque prescriptivo (normas generales) o prestacional (comportamientos individuales), las cuales impiden reconocer condiciones específicas o requieren vastos estudios particulares con los diseños ya elaborados, cuando ya es posible establecer procedimientos intermedios en tipologías y zonas determinadas, aplicando consideraciones generales según modelos paramétricos, para orientar en etapas tempranas del diseño.
Por esta razón, planteamos una codificación por colores en la fase inicial del proyecto arquitectónico que represente desempeños ambientales basados en simulaciones paramétricas de tipologías semejantes implementado en BIM, como una sugerencia gráfica a la configuración en desarrollo, con el fin de motivar mejoramientos ambientales relevantes (de alto impacto y bajo costo) en características perdurables y efectivas del proyecto.

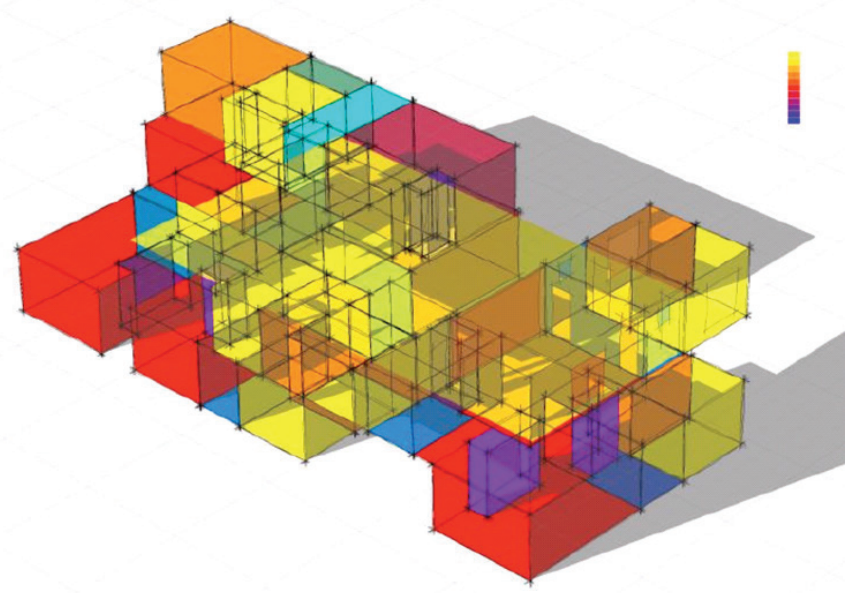

Figura 1: Representación espacial de desempeños 


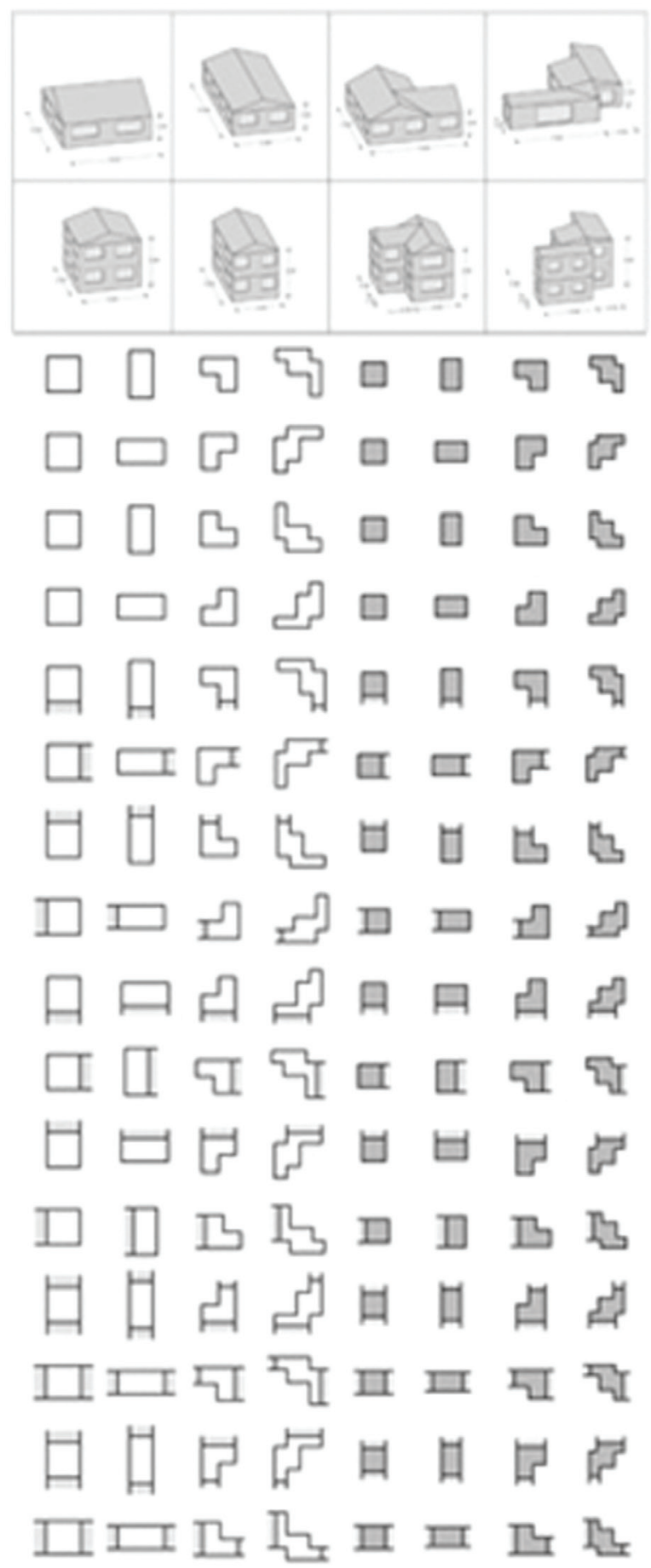

Figura 2: Formas analizadas

\section{Simulaciones Paramétricas}

La estrategia está basada en el cálculo previo de múltiples modelos de un mismo tipo de edificación en una zona determinada, definiendo parámetros formales y constructivos según desempeños ambientales las cuales se implementan como orientaciones tempranas al diseño, sin realizar una simulación completa, pero específicas a edificios de ciertas regiones $y$ funciones.
Esta aproximación se ha experimentado en viviendas unifamiliares del centro-sur de Chile. Esta es una zona de clima templadohúmedo, con variaciones estacionales, que presenta un predominio (sobre un $85 \%$ ) de construcciones residenciales aisladas/pareadas en uno o dos pisos, que combinan paramentos en albañilería y entramados de madera. En magnitudes que varían entre 50 a $250 \mathrm{~m}^{2}$, incluyendo ampliaciones. Con bajos indicadores de confort ; envolvente térmica desmejorada y requerimientos energéticos fundamentalmente de calefacción en invierno.

Aunque se han sugerido diversas relaciones geométricas con el desempeño ambiental de los edificios (denominados usualmente como "factor de forma"), no se han precisado cabalmente y son dependientes de los climas y magnitudes (Olgyay, 1998). Para verificar las condiciones formales de esta tipología se realizaron simulaciones paramétricas de una misma vivienda de $72 \mathrm{~m}^{2}$ en diferentes disposiciones geométricas (fig.2), según el clima de Concepción (el principal centro urbano de la zona), utilizando el Software Ecotect y EnergyPlus, lo que permitió identificar una relación de más del $50 \%$ de la demanda energética para un confort continuo según la longitud de muros expuestos. Obteniendo las configuraciones más compactas, adosadas y bajas, un mejor desempeño (es decir, con menos demanda energética e impacto ambiental), hasta las más extensas; independientes e intrincadas (que requieren mayor demanda energética y situación más variable) lo que coincide con otros estudios (Bustamante 2009; CDT, 2010; Celis, 2012), aunque varían en la incidencia de la altura. Se reconoce también una influencia consistente, aunque leve, de la orientación solar norte, considerando una disposición regular de ventanas.

También se realizaron simulaciones detalladas de una docena de viviendas representativas de la zona, de distintas magnitudes y disposiciones. Se revisaron condiciones geométricas y topológicas de los recintos interiores, según sus desempeños particulares, lo que presenta notables variaciones, que se advierte en la ocupación regular de las casas (habitaciones más frías o temperadas) las que se han monitoreado con sensores térmicos. Definiendo una relación relevante, cercana a la mitad de la demanda, según la proporción de perímetro al exterior de los recintos, lo que es coherente con las condiciones generales de forma. Esto implica que las habitaciones más interiores presentan mejor comportamiento que las dispuestas en los extremos, sin embargo éstas suelen ser preferidas por la mejor iluminación natural, lo que favorece la disposición norte. Se advierte además una vinculación variable con el tamaño de los recintos (aunque se suele percibir que los mayores son más fríos por la lentitud en alcanzar niveles de confort con sistemas de calefacción temporal) y de los niveles (que también se reconoce un mejor desempeño en segundos pisos, por acumulación térmica y mejor asoleamiento).

Estas diferencias entre recintos son relevantes porque los estudios, reglamentaciones y certificaciones, normalmente consideran los edificios de manera global, desconociendo las 
importantes variaciones internas que se producen (y vivimos cada día). Mientras una revisión interior permite asegurar comportamientos integrales.

Las condiciones constructivas también fueron revisadas mediante parámetros de envolvente y renovación de aire según antecedentes previos, evidenciando una incidencia alta y consistente de la transmitancia térmica de los paramentos verticales exteriores, que puede ser regulado por materiales en el desarrollo temprano del diseño, como también, gran influencia de las infiltraciones que depende fundamentalmente de la calidad de ejecución y mantención de la construcción.

Cabe destacar que estas relaciones formales y materiales no son tan directas en zonas climáticas más cálidas que presenten sobrecalentamiento y requieren por tanto extensión y ligereza, así como construcciones de mayor magnitud o complejidad funcional, como los edificios de departamentos, que presentan desempeños más variables.

\section{Visualización Gráfica}

Para expresar estas relaciones de desempeño se plantea una gradiente de colores desde el rojo, que representa sectores de mejor comportamiento (que requieren menos demanda energética y se mantienen naturalmente más cálidos), hasta el azul, que representa elementos con mayor exigencia de energía o más fríos.

Tabla1. Volumen Completo

\begin{tabular}{|c|c|c|c|c|}
\hline Desempeño & $\begin{array}{c}\text { Relación } \\
\text { Per.exp./Sup. } \\
\text { + Sur 25\% }\end{array}$ & Red & Green & Blue \\
\hline Alto & 0,1 & 255 & 0 & 0 \\
\hline Medio & 0,3 & 255 & 0 & 255 \\
\hline Bajo & 0,6 & 0 & 0 & 255 \\
\hline
\end{tabular}

En la forma general de la vivienda (Tabla 1) se considera la relación entre la superficie horizontal total construida interior (en uno o más pisos sumados) y la longitud de muros exteriores. Lo que puede oscilar entre un 0,1 para un volumen más vertical, compacto y adosado que tendría un buen desempeño (baja demanda energética con buen confort continuo), hasta 0,6 o superior, cuando la forma es más extendida, intricada y aislada, y sugiere un bajo desempeño (alta demanda energética y/o débil confort). Con un incremento hacia el Sur sobre un cuarto del perímetro, para expresar la incidencia de menor asoleamiento.

Tabla2. Recintos

\begin{tabular}{|c|c|c|c|c|}
\hline Desempeño & $\begin{array}{c}\text { Relación } \\
\text { Per.exp./Sup. } \\
\mathbf{x} \text { h x Sur }\end{array}$ & Red & Green & Blue \\
\hline Alto & 0 & 255 & 0 & 0 \\
\hline Medio & 0,6 & 255 & 0 & 255 \\
\hline Bajo & 1,2 & 0 & 0 & 255 \\
\hline
\end{tabular}

En los recintos (Tabla 2) se considera la relación entre superficie horizontal continua interior (por piso regular, la mayor altura se incrementa proporcionalmente) y la longitud de muros exteriores (incluyendo pisos elevados o aberturas superiores), lo que puede oscilar desde cero en un recinto central de buen comportamiento ambiental (debidamente ventilado) hasta un recinto rodeado por exterior en la mayoría de sus caras y/o de perfil enmarañado hacia afuera, con un comportamiento más exigente, incrementado parcialmente la longitud hacia el Sur.

Tabla3. Materialidad

\begin{tabular}{|c|c|c|c|c|}
\hline Desempeño & $\begin{array}{c}\text { Transmitancia } \\
\text { global ext. }\end{array}$ & Red & Green & Blue \\
\hline Alto & 0 & 255 & 0 & 0 \\
\hline Medio & 1,0 & 255 & 0 & 255 \\
\hline Bajo & 2,0 & 0 & 0 & 255 \\
\hline
\end{tabular}

Para los elementos perimetrales (muros, pisos, cielo) se considera su transmitancia térmica (valor $\mathrm{U}$ ) ponderada en la superficie total. Regulando por tanto la combinación de materiales o la participación de vanos para motivar la aplicación de mayor aislación térmica, combinado con formas más compactas, y así alcanzar mejores desempeños.

\section{Implementación en BIM}

La representación de estos valores se puede implementar en un software de modelación constructiva (BIM), como Revit de Autodesk, mediante la codificación cromática de sus áreas según las magnitudes geométricas de la envolvente, por ejemplo para expresar la incidencia de la forma general (Fig.3)
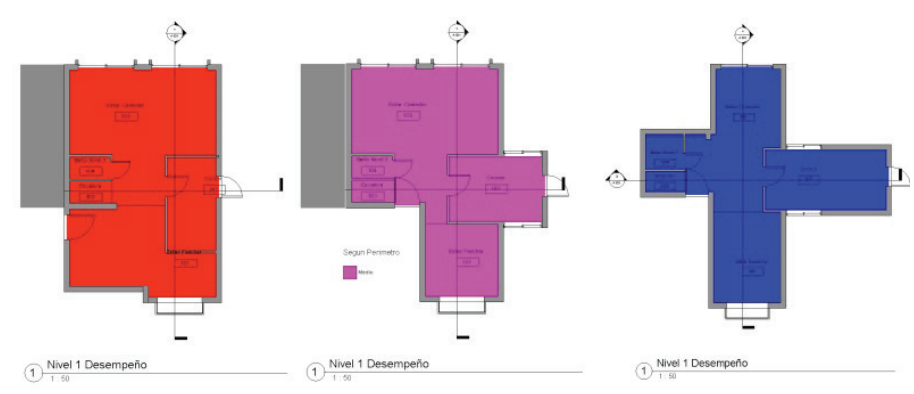

Figura 3: Evolución de Plantas de Vivienda

En las cuales se puede reconocer una variación de color más cálida (rojo) al modificar el diseño con una forma mas compacta y adosada, posible de intervenir con variaciones arquitectónicas de fachada.

De manera similar, en los recintos y elementos constructivos, se puede aplicar esta codificación de colores para fomentar durante el diseño algunas acciones que vayan mejorando el comportamiento ambiental. Por ejemplo, disponiendo recintos más contiguos y regulares con orientación norte y advertir visualmente la incidencia sobre su comportamiento. (Fig.4) 

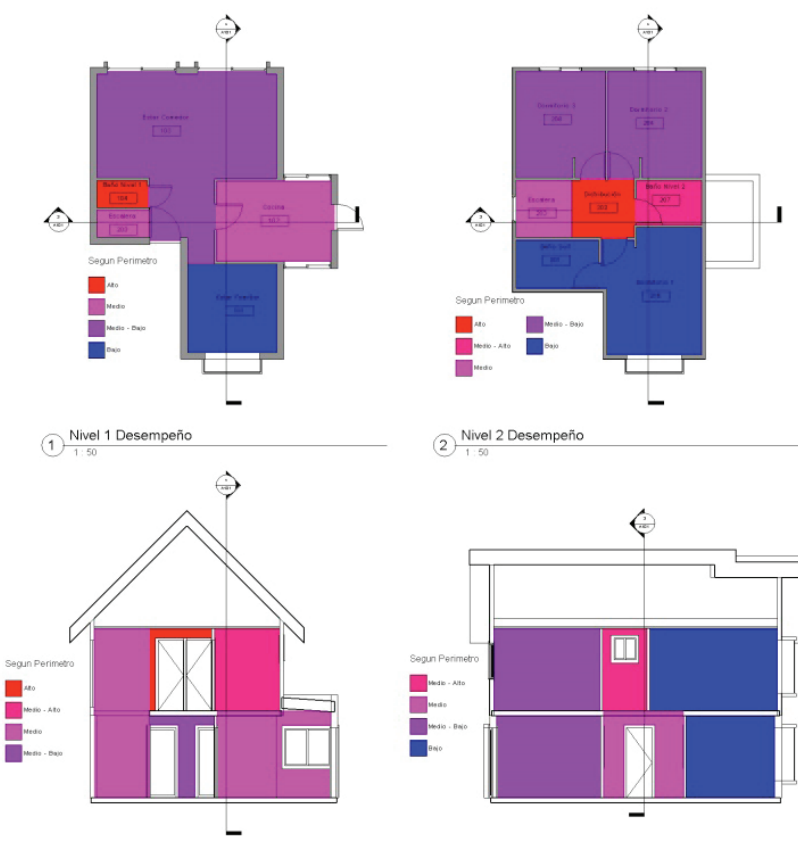

(2) Nivel 2 Desempeño

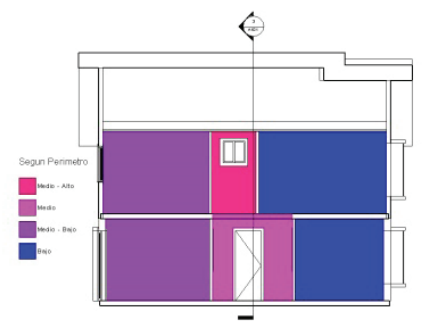

(3) Sección 1

(4) Sección 2

Figura 4: Configuraciones analizadas en Simulaciones Paramétricas

\section{Conclusiones}

Esta estrategia de visualización gráfica en BIM de desempeños basada en simulaciones paramétricas, demuestra la capacidad de integrar recursos computacionales para un desarrollo, exploración y diseño sustentable en etapas tempranas de proyecto, aplicando condiciones tipológicas en la búsqueda de opciones de mejor comportamiento ambiental mediante la combinación de estudios generales y desarrollos particulares. Un avance de esta implementación es una programación interactiva tridimensional (Fig.1), con el fin de fomentar la modificación automática del diseño vinculada con una base de simulaciones (BIM + BPS), estimulando la creatividad sobre regulaciones ambientales efectivas.

\section{Agradecimientos}

Fondecyt 1120165

\section{Referencias}

Morbitzer C., Strachan P., Webster J., Spires B. y Cafferty D. (2001). Integration of Building Simulation into the Design Process of an Architecture Practice, en Seventh International IBPSA Conference, Rio de Janeiro, Brazil, August 13-15, p.697-704

Krieger (2008) Green BIM: Successful Sustainable Design with Building Information Modeling: Steve McDowell, Eddy Krygiel, Brad Nies, Wiley.

Olgyay, V. (1998). Arquitectura y Clima. Barcelona, Ed.G.Gili. 203 p. ISBN : 84-252-1488-2 (trad. de Design with Climate, Princeton, 1963).

Bustamante, W. (2009). Guía de Diseño para la Eficiencia Energética de la Vivienda Social. Santiago, Ministerio de Vivienda y Urbanismo, División Técnica de Estudio y Fomento Habitacional y Programa País de Eficiencia Energética, Colección Monografías y Ensayos (Serie II Tecnología de la Construcción). p. 203

CDT, (2010). Estudio de Usos Finales y Curva de Oferta de Conservación de la Energía en el Sector Residencial. Corporación de Desarrollo Tecnológico: Santiago.

Celis F., Garcia R., Trebilcock M., Escorcia O., Bruscato U. \& Diaz M. (2011). Análisis energético de las viviendas del centro-sur de Chile. Arquiteturarevista, vol. 8, N. 1, p. 62-75 